\title{
White dwarf-main sequence binaries from LAMOST: the DR1 catalogue ${ }^{\star}$
}

\author{
J. J. Ren ${ }^{1,2}$, A. Rebassa-Mansergas ${ }^{3}$, A. L. Luo ${ }^{1,2}$, Y. H. Zhao ${ }^{1,2}$, M. S. Xiang ${ }^{4}$, X. W. Liu ${ }^{3,4}$, \\ G. Zhao ${ }^{1,2}$, G. Jin ${ }^{5}$, and Y. Zhang 6
}

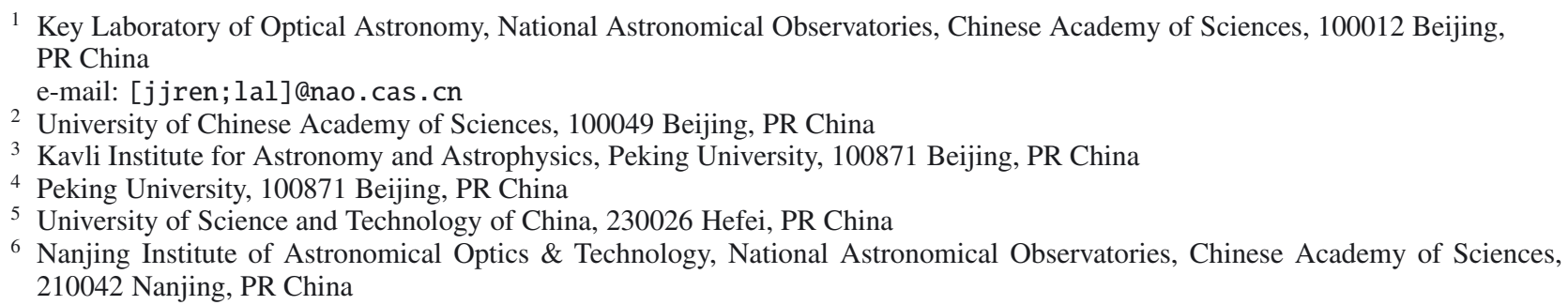

Received 21 February 2014 / Accepted 31 July 2014

\begin{abstract}
Context. White dwarf-main sequence (WDMS) binaries are used to study several different important open problems in modern astrophysics.

Aims. The Sloan Digital Sky Survey (SDSS) identified the largest catalogue of WDMS binaries currently known. However, this sample is seriously affected by selection effects and the population of systems containing cool white dwarfs and early-type companions is under-represented. Here we search for WDMS binaries within the spectroscopic data release 1 of the Large sky Area Multi-Object fiber Spectroscopic Telescope (LAMOST) survey. LAMOST and SDSS follow different target selection algorithms. Hence, LAMOST WDMS binaries may be drawn from a different parent population and thus help in overcoming the selection effects incorporated by SDSS on the current observed population.

Methods. We develop a fast and efficient routine based on the wavelet transform to identify LAMOST WDMS binaries containing a DA white dwarf and a M dwarf companion, and apply a decomposition/fitting routine to their LAMOST spectra to estimate their distances and measure their stellar parameters, namely the white dwarf effective temperatures, surface gravities and masses, and the secondary star spectral types.

Results. We identify 121 LAMOST WDMS binaries, 80 of which are new discoveries, and estimate the sample to be $\sim 90$ per cent complete. The LAMOST and SDSS WDMS binaries are found to be statistically different. However, this result is not due to the different target selection criteria of both surveys, but likely a simple consequence of the different observing conditions. Thus, the LAMOST population is found at considerably shorter distances $(\sim 50-450 \mathrm{pc})$ and is dominated by systems containing early-type companions and hot white dwarfs.

Conclusions. Even though WDMS binaries containing cool white dwarfs are also missed by the LAMOST survey, the LAMOST WDMS binary sample dominated by systems containing early-type companions is an important addition to the current known spectroscopic catalogue. Future LAMOST observations however are required to increase the small number of LAMOST WDMS binaries.
\end{abstract}

Key words. binaries: spectroscopic - stars: low-mass - white dwarfs - methods: data analysis

\section{Introduction}

White dwarf-main sequence (WDMS) binaries are detached compact binary stars formed by a white dwarf and a main sequence star. These binaries evolve from main sequence binaries and can be broadly separated into two groups depending on the current orbital separation (e.g. Farihi et al. 2010). The first group is formed by close WDMS binaries that evolved through a common envelope phase (Iben \& Livio 1993; Webbink 2008). These systems are commonly referred to as post-common envelope binaries or PCEBs and contain $\sim 25$ per cent of the entire WDMS binary population (Willems \& Kolb 2004; Nebot Gómez-Morán et al. 2011). The remaining $\sim 75$ per cent are WDMS binaries

\footnotetext{
* Full Tables 1-3 and the spectra are only available at the CDS via anonymous ftp to cdsarc.u-strasbg. fr (130.79.128.5) or via http://cdsarc.u-strasbg.fr/viz-bin/qcat?]/A+A/570/A107
}

that did not evolve through a common envelope phase. The orbital separation of these systems is roughly the same as the orbital separation of the initial main sequence binary from which they descend.

Thanks to the Sloan Digital Sky Survey (SDSS, York et al. 2000), the number of WDMS binaries has increased dramatically during the last few years (e.g. Silvestri et al. 2007; Heller et al. 2009; Wei et al. 2013). There are currently 2316 WDMS binaries identified within the data release 8 of SDSS (Rebassa-Mansergas et al. 2013a), of which 205 are confirmed as PCEBs (e.g. Schreiber et al. 2010). This catalogue is the largest, most homogeneous and cleanest WDMS binary sample currently available.

The SDSS WDMS binaries and PCEBs are being used to study several different and important aspects in modern astrophysics: e.g. providing constraints on theories of common 
envelope evolution (Davis et al. 2010; Zorotovic et al. 2010; De Marco et al. 2011; Rebassa-Mansergas et al. 2012b; Camacho et al. 2014) and on the origin of low-mass white dwarfs (Rebassa-Mansergas et al. 2011); testing theoretical mass-radius relations of both white dwarfs and low-mass main sequence stars (Nebot Gómez-Morán et al. 2009; Parsons et al. 2010, 2012b,a; Pyrzas et al. 2012); constraining the rotation-ageactivity relation of low-mass main sequence stars (Morgan et al. 2012; Rebassa-Mansergas et al. 2013b); constraining the pairing properties of main sequence stars (Ferrario 2012); probing the existence of circumbinary planets around PCEBs (Zorotovic \& Schreiber 2013; Parsons et al. 2014; Marsh et al. 2014). However, SDSS WDMS binaries suffer from important selection effects (Rebassa-Mansergas et al. 2013a). Namely, the population of SDSS systems containing cool white dwarfs and earlytype companions is clearly under-represented.

In this paper we make use of the recent, multi-faceted and large scale survey operated by Large sky Area Multi-Object fiber Spectroscopic Telescope (LAMOST), also named Guo Shou Jing Telescope to extend the search of WDMS binaries and build-up the current spectroscopic sample. Specifically, we investigate whether or not LAMOST WDMS binaries help in overcoming the selection effects incorporated by SDSS on the current observed population.

\section{The LAMOST survey}

The LAMOST is a quasi-meridian reflecting Schmidt telescope located at Xinglong Observing Station in the Hebei province of China (Cui et al. 2012). It has an effective aperture of about $4 \mathrm{~m}$, and a field of view of $5^{\circ}$ in diameter. The LAMOST is exclusively dedicated to obtain optical spectroscopy of celestial objects. Each spectral plate refers physically to a focal surface with 4000 precisely positioned optical fibers to observe spectroscopic plus calibration targets simultaneously, equally distributed among 16 fiber-fed spectrographs. Each spectrograph is equipped with two CCD cameras of blue and red channels that simultaneously provide blue and red spectra of the 4000 selected targets, respectively.

From 2012 September, LAMOST began its five-year regular survey, before that there was a two-year commissioning survey and a one-year pilot survey. The LAMOST regular survey consists of two main parts (Zhao et al. 2012). The first part is the LAMOST Extra-Galactic Survey (LEGAS) of galaxies to study the large scale structure of the Universe. The second part is the LAMOST Experiment for Galactic Understanding and Exploration (LEGUE) Survey of the Milky Way, developed to study the structure and evolution of the Galaxy (Deng et al. 2012). LEGUE is subdivided into three surveys which follow independent selection criteria for follow-up observations (Carlin et al. 2012; Chen et al. 2012; Liu et al. 2014): the spheroid, the disk, and the galactic anti-centre.

The current data product of LAMOST is data release 1 (DR1). This includes 2204860 spectra, 717660 of which were observed during the pilot survey (Zhao et al. 2012; Luo et al. 2012). The number of spectra classified as star, galaxy, quasar and unknown (mainly due to the poor quality of the spectra) are $1944406,12082,5017$, and 243355 respectively. The resolving power of the LAMOST spectra is $\sim 1800$ and covers the $\sim 3800-9000 \lambda \lambda$ wavelength range. Because of the lack of a network of photometric standard stars, the flux calibration of LAMOST spectra is relative (Song et al. 2012), which means that even though the overall shape of the spectral energy distribution of the targets is well sampled by the flux-calibrated spectra, the level of the absolute flux may not be entirely accurate. In addition, reddening of the standard stars may also affect the flux calibration, especially when the standard stars have very different spatial positions and/or reddening from other stars in the same spectrograph. A considered LAMOST spectrum is unambiguously identified by the MJD (modified Julian date) of the observation, a plate identifier, a spectrograph identifier and a fiber identifier (see Luo et al. 2012, for the description of LAMOST 1D FITS files).

\section{The LAMOST DR1 WDMS binary sample}

In this section we describe in detail our procedure for identifying LAMOST WDMS binaries, estimate the completeness of the identified sample, and evaluate how efficient the selection criteria employed by the LAMOST surveys are in targeting WDMS binaries for follow-up observations.

\subsection{Identification of LAMOST WDMS binaries}

The first catalogue of LAMOST WDMS binaries is provided by Ren et al. (2013) who, based on spectrophotometric colour selection, identified 28 systems from the LAMOST pilot survey. However, the relative flux calibration of the LAMOST spectra (Sect. 2) together with the high Galactic extinction and reddening (Liu et al. 2014, especially in the anti-centre region) incorporate systematic uncertainties to the inferred spectrophotometric magnitudes, which are expected to affect the efficiency of this method in selecting WDMS binaries. An additional technique for identifying WDMS binaries based on $\chi^{2}$ template fitting was developed by Rebassa-Mansergas et al. (2010), which resulted in the largest and most homogeneous catalogue of SDSS WDMS binaries known to date. However, the efficiency of this technique in finding LAMOST WDMS binaries may also be affected by the un-reddened LAMOST spectra, as the $\chi^{2}$ values would increase considerably.

We here developed a novel routine based on the wavelet transform (WT, e.g. Chui 1992) to identify WDMS binaries among the $\sim 2$ million DR1 LAMOST spectra. The WT is an efficient multi-resolution time-frequency analysis tool, which is very popular in many areas of signal processing, and also has been extensively used in astronomical spectral feature extraction (e.g. Starck et al. 1997; Li 2012). The analysis unit of the WT is the local flux of the spectrum, i.e. the selected spectral features. Thus, for a given spectrum, the WT recognises the spectral features rather than the global continuum, and consequently uncertainties in the flux calibration and reddening effects are ignored to some extent. The WT is therefore a suitable method to search for LAMOST WDMS binaries.

In a WT, the considered spectral feature of a spectrum (a WDMS binary spectrum in our case) is decomposed into approximation signals, often referred to as approximation coefficients, and the wavelength values under the considered spectral region are converted into data points. The number of data points is defined mathematically as $\left(N_{2}-N_{1}\right) / 2$, where $N_{2}$ and $N_{1}$ are the superior and inferior sampling data point limits where the WT is applied. The outcome of a WT can be hence considered (for comparative purposes) as a smooth version of the spectrum. This decomposition process can be iterated by decomposing the approximation coefficients into successive approximation coefficients (thus reducing by half the number of data points in each iteration) until the decomposition level is satisfactory. This occurs when the spectral features of a WDMS binary spectrum can be identified in the approximation coefficients as compared to a 
non-WDMS binary spectrum in which the approximation coefficients are dominated by continuum emission and/or by spectral features different from those of WDMS binaries.

Two obvious spectral features can be identified in the spectra of WDMS binaries: the Balmer lines in the blue band (arising from the white dwarf if the atmosphere is hydrogen-rich, i.e. a DA white dwarf) and the molecular absorption bands in the red band (typical features of late type, M dwarf companions). We thus selected the 3910-4422 $\lambda \lambda$ range in the blue band (which includes the $\mathrm{H} \gamma, \mathrm{H} \delta$ and $\mathrm{H} \epsilon$ Balmer lines) and the 6800$8496 \lambda \lambda$ range in the red band (which samples a large number of $\mathrm{TiO}$ and VO molecular bands) as the spectral regions to perform the WT. This obviously limited our search to WDMS binaries containing a DA white dwarf and a M dwarf companion, hereafter DA/M binaries. However, reliable stellar parameters (fundamental for characterising the LAMOST population) can only be obtained for DA/M binaries (Rebassa-Mansergas et al. 2010). Moreover, DA/M binaries are the most common ( $~ 80$ per cent) among WDMS binaries (Rebassa-Mansergas et al. 2013a).

We determined the appropriate number of WT iterations to be performed to the two selected spectral regions as followed. First, we visually inspected all spectra classified as white dwarf, A star, K star, M star and Double (i.e. binary star) by the LAMOST pipelines and identified 82 clear DA/M binary spectra, a list we refer to as the test sample hereafter. We note that we inspected these spectral type groups simply because DA/M binaries are likely typed as such by the LAMOST pipelines. Then, we randomly added a large number of spectra classified as Galaxy, quasar, and star (spectral type O, B, A, F, G, K, M and white dwarf; which are not DA/M binaries) to this list. Finally, we applied successive WT iterations to all spectra in our test sample and evaluated when the approximation coefficients became blurred or unrecognisable for our DA/M binaries. We found this happened for the majority of cases at the 6th iteration in the blue band and the 8th iteration in the red band. We therefore fixed the number of iterations as five and seven in the blue and the red bands, respectively. This corresponds to 18 data number points left after the 5 th iteration in the blue band and 14 data points left after the 7th iteration in the red band. In Fig. 1 we show the approximation coefficients as a function of data number of points obtained for the successive WT iterations applied to a DA/M binary (top left), a M star (top right), an A star (bottom left) and a $\mathrm{G}$ star (bottom right) in our test sample. It becomes obvious that the approximation coefficients of the A star and the DA/M binary are similar in the blue band, the same as the approximation coefficients of the M star and the DA/M binary in the red band. Therefore, in order to efficiently select DA/M binaries against other types of celestial objects we applied the following cuts in the blue band and red band,

$$
\begin{aligned}
& f_{\mathrm{b}}\left[i_{1}\right]<\max \left(f_{\mathrm{b}}\left[i_{1}+1\right], f_{\mathrm{b}}\left[i_{1}+2\right]\right) \| \\
& \left(f_{\mathrm{b}}\left[i_{1}\right]<f_{\mathrm{b}}\left[i_{1}+2\right] \& f_{\mathrm{b}}\left[i_{1}\right]<\max \left(f_{\mathrm{b}}\left[i_{1}-1\right], f_{\mathrm{b}}\left[i_{1}-2\right]\right)\right) \& \\
& \left(f_{\mathrm{b}}\left[i_{2}\right]<f_{\mathrm{b}}\left[i_{2}-2\right] \& f_{\mathrm{b}}\left[i_{2}\right]<\max \left(f_{\mathrm{b}}\left[i_{2}+2\right], f_{\mathrm{b}}\left[i_{2}+3\right]\right)\right) \& \\
& \left(f_{\mathrm{b}}\left[i_{3}\right]<f_{\mathrm{b}}\left[i_{3}-3\right] \& f_{\mathrm{b}}\left[i_{3}\right]<f_{\mathrm{b}}\left[i_{3}+1\right]\right)
\end{aligned}
$$

and

$$
\begin{aligned}
& f_{\mathrm{r}}\left[j_{1}\right]>f_{\mathrm{r}}\left[j_{1}+1\right] \&\left(f_{\mathrm{r}}\left[j_{2}\right]>f_{\mathrm{r}}\left[j_{2}-3\right] \&\right. \\
& \left.f_{\mathrm{r}}\left[j_{2}\right]>f_{\mathrm{r}}\left[j_{2}+2\right]\right) \& \min \left(f_{\mathrm{r}}\left[j_{3}\right], f_{\mathrm{r}}\left[j_{3}-1\right]\right)< \\
& \max \left(f_{\mathrm{r}}\left[j_{3}-2\right], f_{\mathrm{r}}\left[j_{3}-3\right], f_{\mathrm{r}}\left[j_{3}-4\right]\right)
\end{aligned}
$$

respectively, where $f_{\mathrm{b}}$ and $f_{\mathrm{r}}$ are the approximation coefficients in the blue and the red bands respectively at the corresponding data number $i$ and $j$, and $i_{1}=3, i_{2}=7, i_{3}=15$, and $j_{1}=1, j_{2}=5, j_{3}=13$. In the blue band these equations select the DA/M binary and the A star shown in Fig. 1 and exclude the G star and the M star, whilst in the red band they select the DA/M binary and the M star but exclude the A star and the $\mathrm{G}$ star. Thus, only the DA/M binary survives the applied cuts in Fig. 1. From the complete test sample, these cuts efficiently selected 79 of the $82 \mathrm{DA} / \mathrm{M}$ binaries. However, we note that we applied a large number of different cuts, and only those provided here resulted in the largest number of DA/M binaries identified from our test sample.

Applying the WT and above define cuts to all $\sim 2$ million DR1 LAMOST spectra resulted in a sample of 8543 selected spectra, which we visually inspected. This resulted in the identification of 130 genuine DA/M binary spectra of 118 unique systems (nine objects were observed twice by LAMOST, and one was observed four times). To this list we added the three $\mathrm{DA} / \mathrm{M}$ binary spectra missed from the test sample (one is a different spectrum of a DA/M binary already identified), thus increasing the total number of LAMOST DA/M binaries to 120 (133 total spectra). A cross-correlation of our list of DA/M binaries with the catalogue by Rebassa-Mansergas et al. (2013a) revealed that 41 systems were previously observed by SDSS, which implies we have identified 79 new systems in this work. We also find a large number (180) of systems displaying both a red and a blue component in the LAMOST spectra, but that we do not consider as DA/M binaries. The spectra of these objects are either too noisy, or are likely to be the result of the superposition of two stars in the line of sight, or the result of white dwarfs (M-dwarfs) located close to very bright M-dwarfs (white dwarfs or A-stars) causing scattered light to enter the spectroscopic fiber, i.e. an apparent two-component spectrum.

Finally, we compared our list of 120 systems with the sample of 28 LAMOST DA/M binaries from the pilot survey of LAMOST (Ren et al. 2013). Our WT method finds 26 of the 28 listed targets. However, four of these (J052529.17+283705.4，J052531.26+283807.6， J052531.33+ 284549.4, and J120024.56+292310.3) are not included in our sample as we do not consider them DA/M binaries. Of the two objects we missed, one $(\mathrm{J} 132417.76+280755.8)$ is a DA/M and we add it to our list, the other (J150626.53+275925.2) a DA/M binary candidate. The total number of LAMOST DA/M binaries thus increases to 121 (the total number of LAMOST spectra is 134) and the final number of new DA/M binaries found in this work, i.e. systems that were not observed by SDSS, increases to 80. Table 1 lists all our LAMOST DA/M binaries. For completeness, we also include in this list the 181 systems displaying two components in the LAMOST spectra but that are not considered as DA/M binaries by us. Table 2 lists the SDSS ugriz or Xuyi gri magnitudes and coordinates of our 121 DA/M binaries (the Xuyi survey is the photometric counterpart of the LAMOST survey of the Galactic anti-centre; see Sect. 2). In Fig. 2 we show the LAMOST spectra of $12 \mathrm{DA} / \mathrm{M}$ binaries in our catalogue (the complete catalogue is available at the CDS).

\subsection{The efficiency of the wavelet transform method and completeness of the LAMOST WDMS binary sample}

In order to evaluate the efficiency of the WT method in finding DA/M binaries as well as to estimate the completeness of the LAMOST DA/M binary sample, i.e. the fraction of DA/M binaries we identified respect to the total number observed, we applied the WT method to all $\sim 1.8$ million SDSS DR 8 spectra. Our purpose here is to check how many of the 1819 SDSS DR 8 

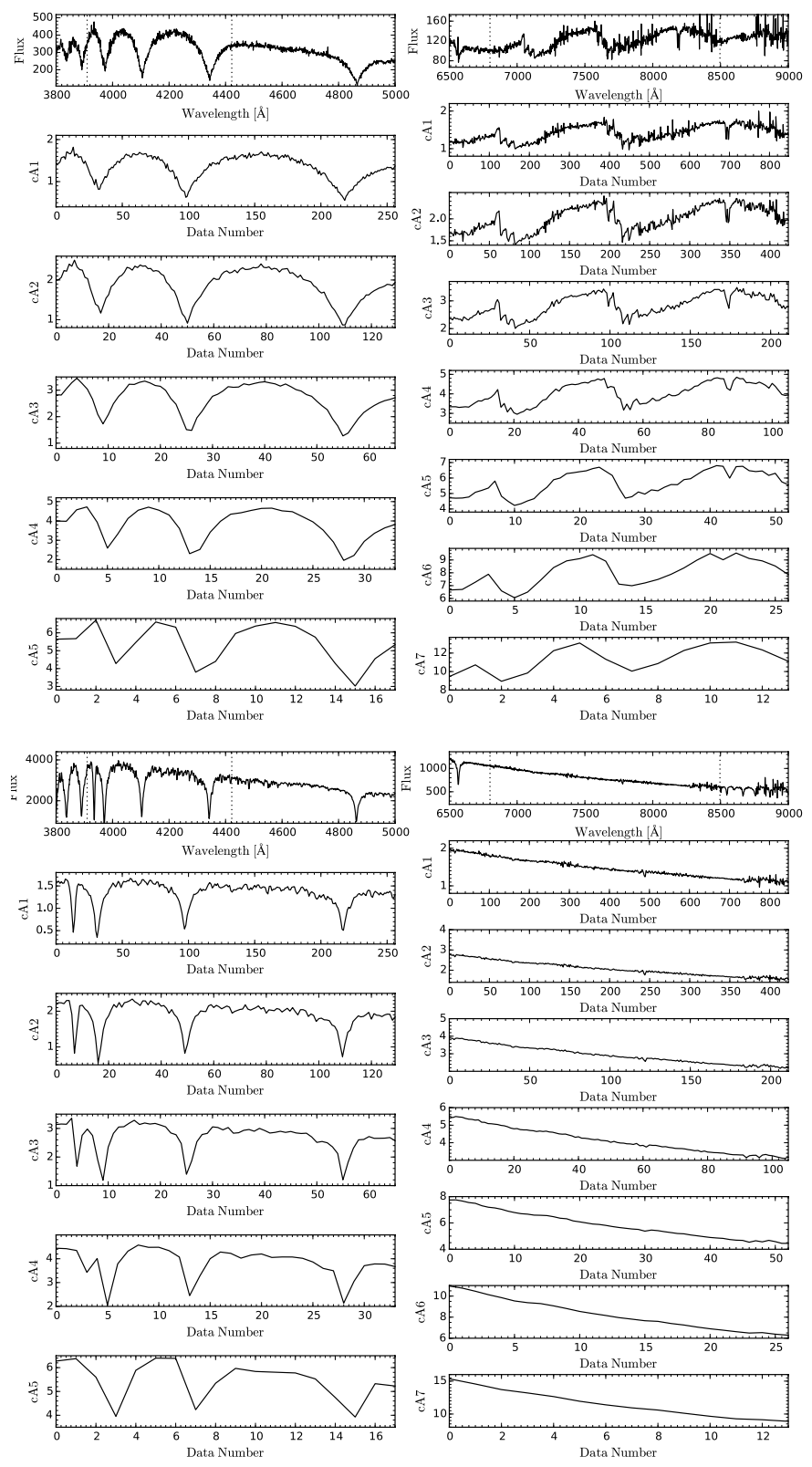
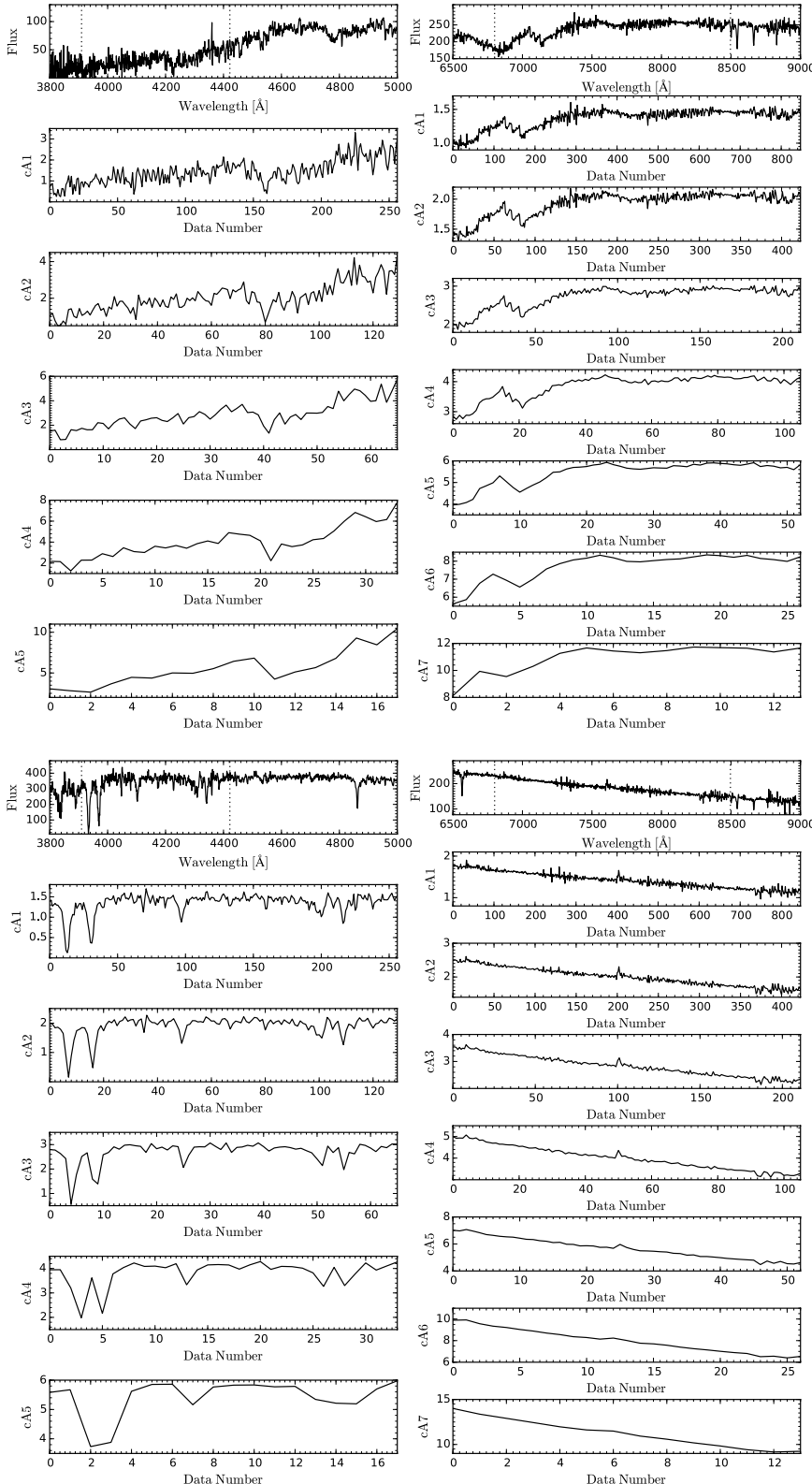

Fig. 1. Approximation coefficients (CA) as a function of data number obtained applying the wavelet transform to a LAMOST DA/M binary (J075919.43+321948.1; top left), a M star (top right panels), an A star (bottom left panels), and a G star (bottom right panels). The LAMOST spectra of each target are shown in the top panels (flux units are $10^{-17} \mathrm{erg} / \mathrm{cm}^{-2} \mathrm{~s}^{-1} \AA^{-1}$ ), where the vertical grey dotted lines define the spectral regions selected to perform the wavelet transform.

DA/M binaries (Rebassa-Mansergas et al. 2013a) can be identified by the WT routine. After applying the WT to all SDSS DR 8 spectra we obtained 27060 WDMS binary candidate spectra, and after a visual inspection we identified a total of 1646 systems as DA/M binaries. Therefore, the WT recovered $\sim 90$ per cent of the SDSS DA/M binary sample. Given that the SDSS WDMS binary catalogue is $\gtrsim 98$ per cent complete (Rebassa-Mansergas et al. 2013a), the exercise performed here strongly suggests the WT should select also $\sim 90$ per cent of all DA/M binaries observed by LAMOST. The spectra of the 173 SDSS DR8 DA/M binaries the WT failed to identify miss important data points in the areas in which the WT is applied, or are clearly dominated by one of the two stellar components, or are spectra of extremely low signal-to-noise ratio $(\mathrm{S} / \mathrm{N})$ for a clear identification of the spectral features of the two stars. We expect the $\sim 10$ per cent of LAMOST DA/M binary spectra missed by the WT to be of the same characteristics.
We conclude that the WT is an efficient method for identifying DA/M binaries and that the completeness of the LAMOST $\mathrm{DA} / \mathrm{M}$ binary sample is $\sim 90$ per cent.

\subsection{The efficiency of the LAMOST target selection algorithm in selecting WDMS binaries}

The number of spectra released by SDSS DR 8 ( $\sim 1.8$ million) and LAMOST DR 1 ( $\sim 2$ million) are similar. However, the total number of DA/M binaries in LAMOST DR 1 (121) is more than one order of magnitude lower than in SDSS DR 8 (1819). This difference is very likely a consequence of the different target selection criteria employed by the two surveys. We investigate this hypothesis here.

In Fig. 3 (left panel) we show the $u-g$ vs. $g-r$ density map for 497354 SDSS DR 8 sources with available spectra, clean photometry, and photometric errors of less than 0.05 mag. 
Table 1. Our catalogue of 121 LAMOST DA/M binaries.

\begin{tabular}{|c|c|c|c|c|c|c|c|c|c|c|c|c|c|c|c|}
\hline Jname & $S / N$ & MJD & Plateid & Spid & Fiberid & $\begin{array}{l}T_{\text {eff }}^{\mathrm{wd}} \\
{[\mathrm{K}]}\end{array}$ & Err & $\log (g)_{\mathrm{wd}}$ & Err & $\begin{array}{c}M_{\mathrm{wd}} \\
{\left[M_{\odot}\right]}\end{array}$ & Err & $\begin{array}{c}d \\
{[\mathrm{pc}]}\end{array}$ & Err & $\mathrm{Sp}$ & Type \\
\hline J002237.90+334322.1 & 5.48 & 56255 & VB007N33V1 & 03 & 085 & 0 & 0 & 0 & 0 & 0 & 0 & 0 & 0 & -1 & 0 \\
\hline $52.22+$ & 37.36 & 56266 & M31007N & & 196 & 34926 & 1011 & 8.29 & 0.19 & 0.83 & 0.11 & 245 & 34 & 2 & 0 \\
\hline J004232.56+415403.0 & 8.75 & 55886 & M31_004437N404045_M2 & 15 & 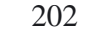 & 15071 & 702 & 7.08 & 0.16 & 0.26 & 0.04 & 238 & 27 & 6 & 0 \\
\hline J004432.63+402635.2 & 11.25 & 55886 & M31_004437N404045_M2 & 04 & 127 & 0 & 0 & 0 & 0 & 0 & 0 & 0 & 0 & 3 & 0 \\
\hline J004545.96+415029.9 & 4.57 & 55863 & M31_011N40_B1 & 15 & 128 & 0 & 0 & 0 & 0 & 0 & 0 & 0 & 0 & 2 & 0 \\
\hline J005221.55+440335.4 & 15.87 & 56207 & M31011N44B1 & 08 & 149 & 22292 & 3046 & 8.23 & 0.48 & 0.77 & 0.29 & 225 & 74 & 2 & 0 \\
\hline J005601.44+400718.4 & 28.14 & 55886 & M31_004437N404045_M2 & 06 & 151 & 0 & 0 & 0 & 0 & 0 & 0 & 0 & 0 & 2 & 0 \\
\hline J005636.91+383332.6 & 16.69 & 55914 & M31_01h38_F1 & 03 & 098 & 0 & 0 & 0 & 0 & 0 & 0 & 0 & 0 & 4 & 0 \\
\hline J005853.40+064849.3 & 59.70 & 56240 & EG010249N073002F & 03 & 027 & 11565 & 247 & 7.55 & 0.25 & 0.38 & 0.11 & 229 & 35 & 1 & 0 \\
\hline $\mathrm{J} 011447.51+254229.6$ & 16.66 & 56267 & EG011927N271550M01 & 02 & 241 & 35740 & 628 & 7.81 & 0.12 & 0.57 & 0.06 & 140 & 12 & 1 & 0 \\
\hline
\end{tabular}

Notes. White dwarf stellar parameters (effective temperature, surface gravity and mass), spectral type of the companions and distances are given when available, however only those with a $\mathrm{S} / \mathrm{N}$ higher than 12 (second column) are considered in the analysis of this paper. Spectral types of -1 imply that no values are available. Modified Julian date (MJD), plate (plateid), spectrograph (spid) and fiber (fiberid) identifiers for each of the LAMOST spectra are also provided. For completeness, we also include 181 systems that are not considered by us as DA/M binaries but that show blue and red components in their spectra. These are flagged as 1 in the last column. The complete table can be found at the CDS.

Table 2. SDSS or Xuyi magnitudes (when available) and coordinates of our 121 LAMOST DA/M binaries.

\begin{tabular}{ccccccccc}
\hline \hline Jname & RA [deg] & Dec [deg] & $u$ & $g$ & $r$ & $i$ & $z$ & SDSS/Xuyi \\
\hline J002237.90+334322.1 & 5.65794 & 33.72280 & 19.56 & 17.35 & 15.98 & 14.58 & 13.87 & SDSS \\
J002952.22+434205.8 & 7.46761 & 43.70163 & 0 & 0 & 0 & 0 & 0 & 0 \\
J004232.56+415403.0 & 10.63567 & 41.90083 & 17.35 & 17.18 & 17.43 & 17.40 & 17.09 & SDSS \\
J004432.63+402635.2 & 11.13595 & 40.44311 & 19.80 & 19.03 & 17.96 & 16.77 & 16.08 & SDSS \\
J004545.96+415029.9 & 11.44152 & 41.84165 & 0 & 0 & 0 & 0 & 0 & 0 \\
J005221.55+440335.4 & 13.08981 & 44.05985 & 17.05 & 16.46 & 15.62 & 14.61 & 13.95 & SDSS \\
J005601.44+400718.4 & 14.00604 & 40.12179 & 18.81 & 18.13 & 17.23 & 16.35 & 15.88 & SDSS \\
J005636.91+383332.6 & 14.15381 & 38.55906 & 0 & 0 & 0 & 0 & 0 & 0 \\
J005853.40+064849.3 & 14.72252 & 6.81371 & 17.30 & 17.18 & 17.46 & 17.64 & 17.73 & SDSS \\
J011447.51+254229.6 & 18.69799 & 25.70823 & 16.96 & 17.18 & 17.49 & 17.31 & 16.85 & SDSS \\
\hline
\end{tabular}

Notes. The complete table can be found at the CDS.

In the right panel of the same Figure we represent the same density map for 587988 DR 1 LAMOST targets with available SDSS DR 8 ugr magnitudes, where again we select only objects with clean photometry and photometric errors below 0.05 . We used this particular colour diagram because single main sequence stars can be easily excluded in the $u-g$ vs. $g-r$ plane, which allows us to analyse the impact of the selection criteria in targeting DA/M binaries in both quasar dominated and quasar free colour areas by the two surveys. The colour cut defined by Rebassa-Mansergas et al. (2013a) for excluding single main sequence stars is illustrated as black solid lines in both panels of Fig. 3, together with 1523 SDSS DR 8 and 70 LAMOST DR 1 $\mathrm{DA} / \mathrm{M}$ binaries that satisfy the above photometric conditions (red open circles). For comparative purposes, SEGUE (the SDSS Extension for Galactic Understanding and Exploration, Yanny et al. 2009) DA/M binaries, which contain systematically cooler white dwarfs and earlier type secondaries (Rebassa-Mansergas et al. 2012a), are displayed as open blue circles in the left panel of Fig. 3. It is worth noting that the LAMOST survey of the Galactic anti-centre, i.e. the GAC survey (Sect. 2), does not overlap with SDSS DR 8, therefore the vast majority of targets observed are not included in Fig. 3 because of lack of $u g r$ magnitudes. Thus, we use the $g-r$ colours obtained from Xuyi photometry (the photometric counterpart of the GAC survey, Liu et al. 2014) of all LAMOST DA/M binaries that were observed as part of the GAC survey and illustrate them as blue open circles in the right panel of Fig. 3, where we assume a $u-g$ colour of 0 because of the lack of Xuyi $u$-band photometry.
Inspection of Fig. 3 reveals that outside the DA/M binary colour space area the LAMOST and SDSS density maps are rather similar, the only difference being that LAMOST observed a considerably larger number of main sequence stars. Within the DA/M binary box it becomes clear that SDSS observed approximately half an order of magnitude more sources than LAMOST. This can clearly be seen in the number distribution of targets as a function of $u-g$ (top panels) and $g-r$ (right panels), and has an easy explanation: whilst one of the main drivers of SDSS has been targeting galaxies and quasars for spectroscopy (Strauss et al. 2002; Richards et al. 2002; Adelman-McCarthy et al. 2008) (see the prominent peaks at $g-r \simeq 0.2$ and $u-g \simeq 0.4$ ), LAMOST is a dedicated Galactic survey targeting mainly stars (Zhao et al. 2012, see also Sect. 2). It is therefore not surprising that the number of DA/M binaries observed by SDSS is considerably larger than the number observed by LAMOST.

Independently of the total number of DA/M binaries observed by the two surveys, a different and more important issue in the context of this paper is whether the LAMOST DA/M binary population has different properties than the one observed by SDSS. Specifically, whether or not LAMOST DA/M binaries contain systematically cooler white dwarfs and earlier-type companions, a population under-represented in the SDSS sample. Although we will investigate this in detail in Sect. 5, it is worth noting that $\sim 70$ per cent of SDSS and $\sim 60$ per cent of LAMOST DA/M binaries are concentrated in quasar dominated colour areas $(g-r \lesssim 0.7)$ representative of systems containing hot white dwarfs (open circles in both panels of Fig. 3). As 

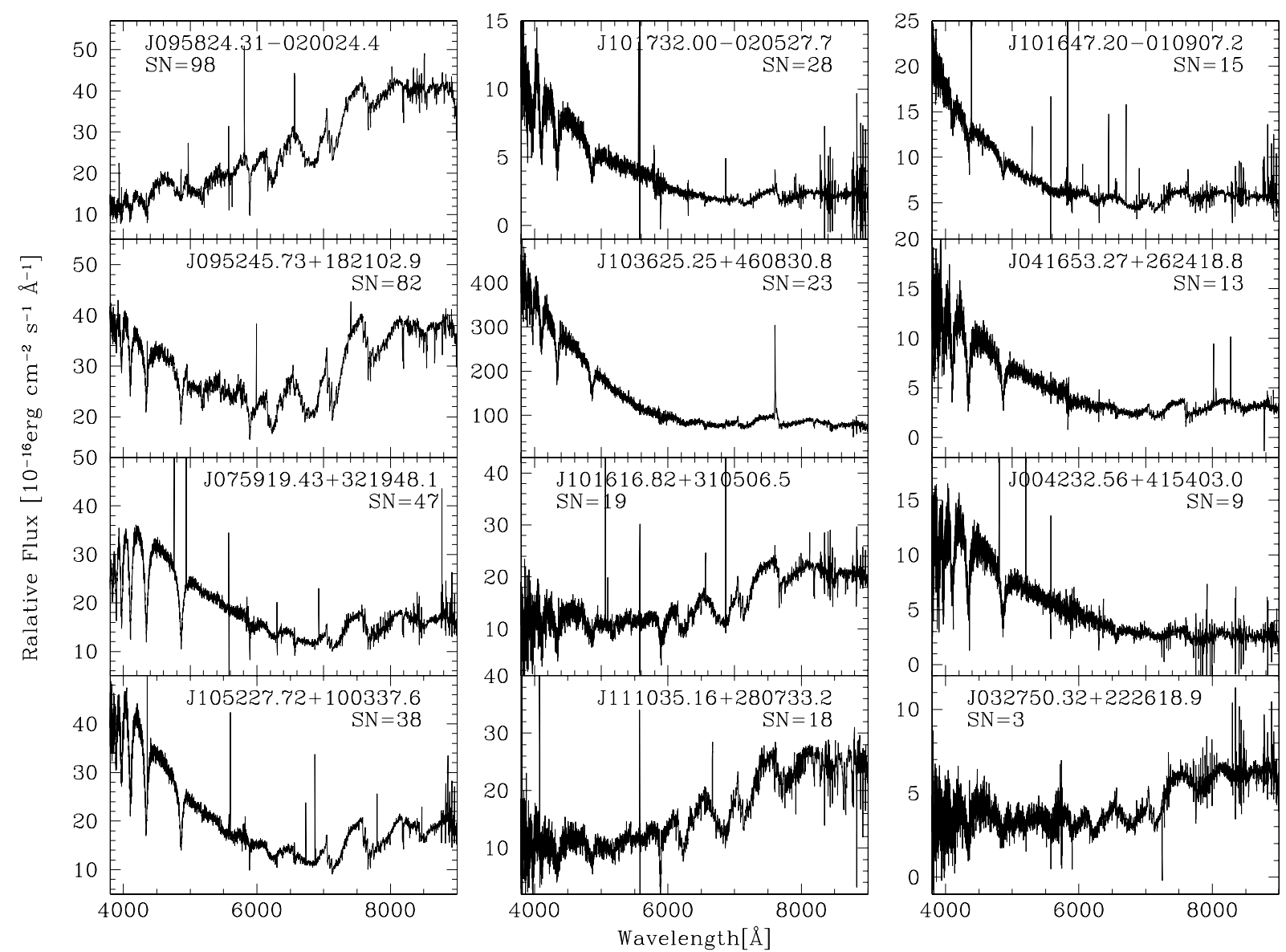

Fig. 2. Example spectra of 12 LAMOST DA/M binaries, ordered in appearance according to the S/N of the LAMOST spectra. The complete catalogue is available at the CDS.
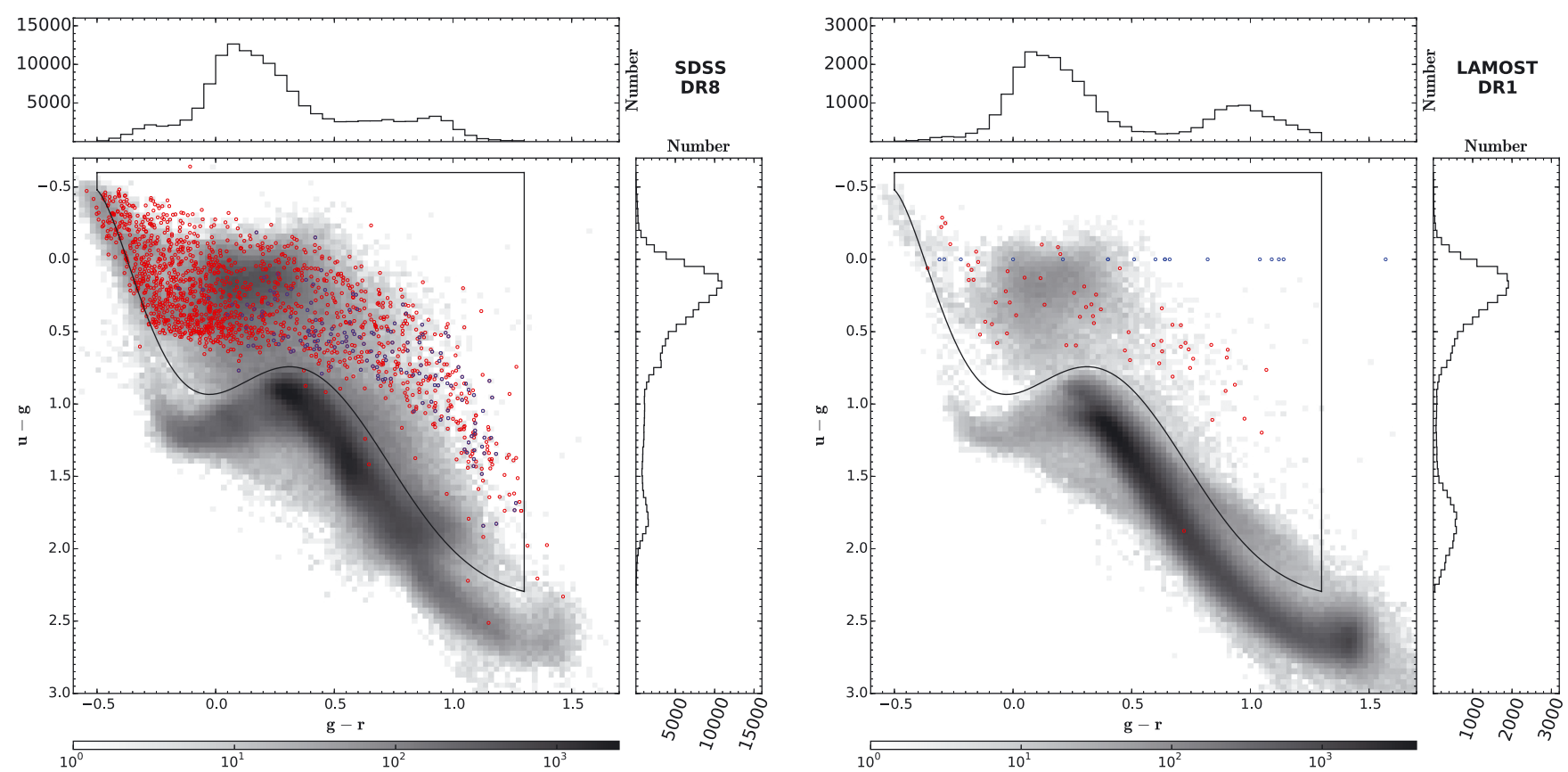

Fig. 3. SDSS (left) and LAMOST (right) density maps in the $u-g$ vs. $g-r$ plane. Identified DA/M binaries are shown as red open circles (blue open circles represent SEGUE and GAC DA/M binaries in the left and right panels, respectively). The solid black lines are colour-cuts defined for selecting DA/M binaries and excluding single main sequence stars. The number distribution of objects as a function of $u-g$ and $g-r$ within these cuts are provided in the upper and right panels, respectively. 

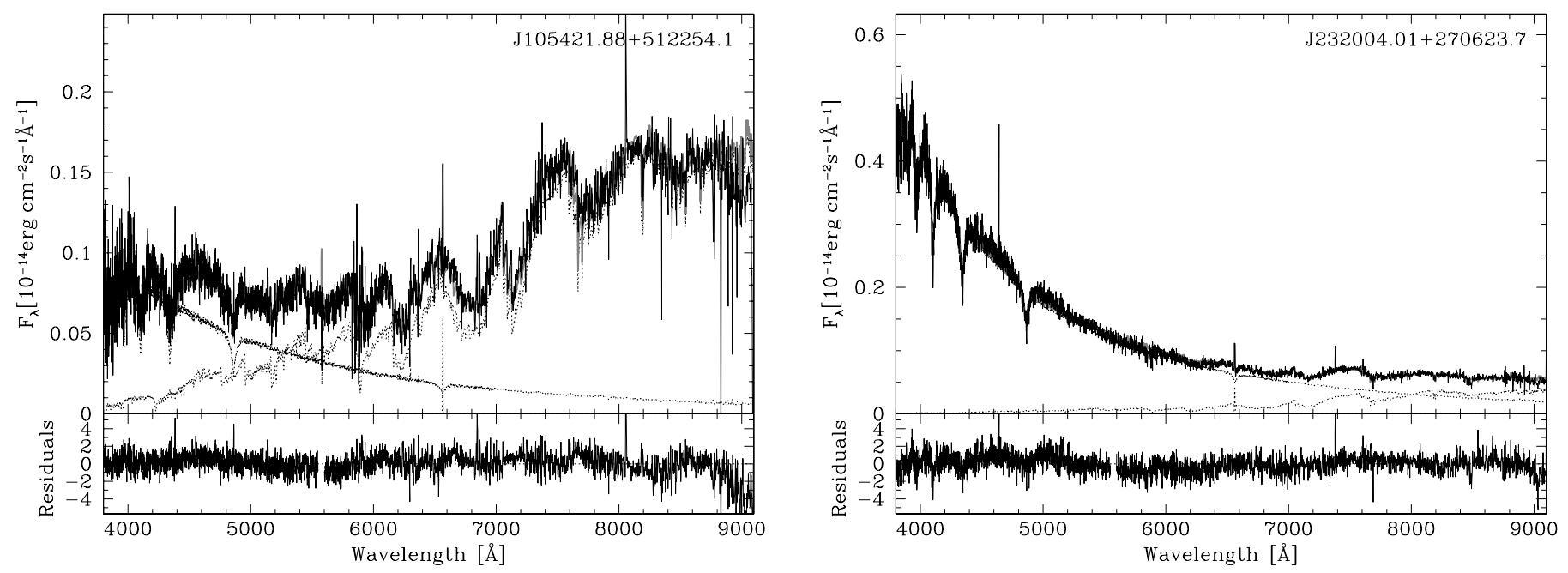

Fig. 4. Two-component fits to the LAMOST DA/M binary spectra. Shown are examples for objects with either the M-dwarf or the white dwarf dominating the LAMOST spectrum. Top panels: DA/M binary spectrum as black line, and the two templates, white dwarf and M-dwarf, as dotted lines. Bottom panels: residuals from the fit.

expected, SEGUE DA/M binaries (blue open circles; left panel of Fig. 3) which are intrinsically dominated by cooler white dwarfs and earlier-type companions, generally populate colour areas that do not overlap with those of quasars $(g-r \gtrsim 0.7)$. Interestingly, this is also the case for $\sim 50$ per cent of LAMOST $\mathrm{DA} / \mathrm{M}$ binaries observed by the GAC survey (blue open circles in Fig. 3, right panel). Therefore the subpopulation of LAMOST GAC DA/M binaries may deserve further attention.

We conclude that the low number of DA/M binaries observed by LAMOST as compared to SDSS is a simple consequence of the differences in the target selection criteria of both surveys. We also find strong indications for a large population of LAMOST DA/M binaries containing hot white dwarfs, which indicates the LAMOST surveys may not help in overcoming the selection effects incorporated by SDSS. We will discuss this in detail in Sect. 5.

\section{Stellar parameters and distances}

Reddening is likely to affect the flux calibration of LAMOST spectra (Sect. 2), which may affect the determination of the stellar parameters of our DA/M binaries (especially if these are located in the Galactic anti-centre region). To solve this potential problem we re-flux calibrated the spectra of our 121 targets following the routine developed by Xiang et al. (2014). This method makes use of de-reddened flux standard stars observed in the same spectrograph as the considered targets to provide de-reddened and flux calibrated target spectra. However, the flux calibration applied by the Xiang et al. (2014) routine is also relative, i.e. the overall shape of the spectral energy distribution is well sampled by the flux-calibrated spectra but the absolute flux level may not be entirely accurate.

We applied the decomposition/fitting routine of Rebassa-Mansergas et al. (2007) to the re-calibrated spectra for measuring the stellar parameters and estimating the distances to our DA/M binaries. This method is briefly described as followed. First, a given LAMOST DA/M binary spectrum is fitted with a two-component model using a set of observed $\mathrm{M}$ dwarf and white dwarf templates. From the converged fit to each spectrum (see Fig. 4) we record the spectral type of the secondary star. The best-fit M dwarf template is then subtracted and we fit the residual white dwarf spectrum with a model grid of DA white dwarfs (Koester 2010) to obtain its effective temperature, $T_{\text {eff }}$, and surface gravity, $\log g$ (see Fig. 5). The equivalent widths of the Balmer lines go through a maximum near $T_{\text {eff }}=13000 \mathrm{~K}$, with the exact value being a function of $\log g$. Therefore, $T_{\text {eff }}$ and $\log g$ determined from Balmer line profile fits are subject to an ambiguity, often referred to as "hot" and "cold" solutions, i.e. fits of similar quality can be achieved on either side of the temperature at which the maximum equivalent width is occurring. We use the $T_{\text {eff }}$ and $\log g$ obtained from the fits to the whole spectrum, continuum plus lines (excluding the region above $7000 \AA$, where residual contamination from the secondary star subtraction is largest) to select the "hot" or "cold" solution from the line profile fits. The mass and the radius of the white dwarf is then obtained interpolating the determined $T_{\text {eff }}$ and $\log g$ in the tables provided by Bergeron et al. (1995) ${ }^{1}$. However, it has been shown that one-dimensional white dwarf model spectra such as those used in this work yield overestimated $\log g$ (and therefore mass) values for white dwarfs of effective temperatures below $\sim 12000 \mathrm{~K}$ (e.g. Koester et al. 2009; Tremblay et al. 2011). Therefore, we apply the corrections of Tremblay et al. (2013), which are based on three-dimensional white dwarf model spectra, to our white dwarf parameter determinations. Finally, we use the flux scaling factors between the observed residual white dwarf spectra and the best-fit white dwarf models to estimate the white dwarf distances, which are equivalent to the distances to our DA/M binaries ${ }^{2}$. It is important to mention that the relative flux calibration of the LAMOST spectra may incorporate systematic uncertainties in the distance determinations, as the flux scaling factor depends on the absolute level of the flux-calibrated spectra, and we will discuss this effect in detail below. Table 1 provides the

\footnotetext{
1 Updated tables can be found at http://www . astro.umontreal. $\mathrm{ca} /$ bergeron/CoolingModels/

2 In principle, we could also get a distance estimate from the flux scaling factor between the observed spectra and best-fit templates to the secondary stars. However, it has been shown that $\sim 1 / 3$ of the secondary star distances obtained in this way are systematically larger than the distances measured to the white dwarf components, presumably due to magnetic activity (Rebassa-Mansergas et al. 2007). Therefore we do not take into account the secondary star distances in this work.
} 

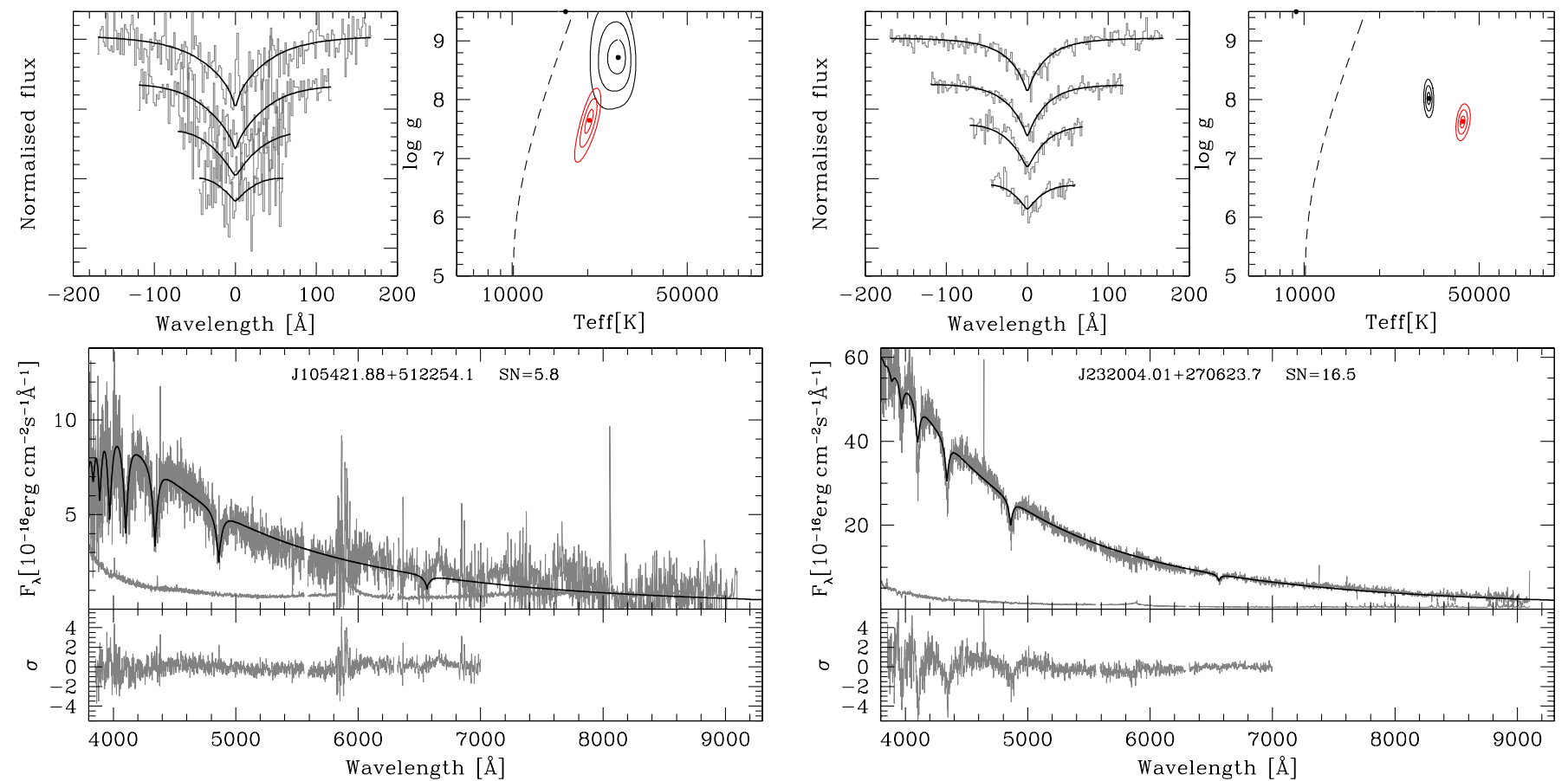

Fig. 5. Spectral model fits to the white dwarf components of the two DA/M binaries shown in Fig. 4, obtained after subtracting the best-fit M-dwarf template. Top left panels: best-fit (black lines) to the normalised $\mathrm{H} \beta$ to $\mathrm{H} \epsilon$ (grey lines, top to bottom) line profiles. Top right panels: 3 , 5 , and $10 \sigma$ $\chi^{2}$ contour plots in the $T_{\text {eff }}-\log g$ plane. The black contours refer to the best line profile fit, the red contours to the fit of the whole spectrum. The dashed line indicates the occurrence of maximum $\mathrm{H} \beta$ equivalent width. The best "hot" and "cold" line profile solutions are indicated by black dots, the best fit to the whole spectrum is indicated by a red dot. Bottom panels: residual white dwarf spectra resulting from the spectral decomposition and their flux errors (grey lines) along with the best-fit white dwarf model (black line) to the 3800-7000 $\lambda \lambda$ wavelength range (top) and the residuals of the fit (grey line, bottom). The $T_{\text {eff }}$ and $\log g$ values listed in Table 1 are determined from the best line profile fit. The fit to the whole spectrum is only used to select between the "hot" and "cold" line fit.

white dwarf stellar parameters of $65 \mathrm{DA} / \mathrm{M}$ binaries, and the $\mathrm{M}$ dwarf spectral types of $104 \mathrm{DA} / \mathrm{M}$ binaries. The LAMOST spectra of the DA/M binaries for which we could not obtain stellar parameters are either too noisy, are dominated by the flux from one of the two stellar components, or are subject to a bad flux calibration due to lack of suitable flux standard stars. Because of these reasons we are able to obtain white dwarf stellar parameters and spectral types of the companions for 27 and 40 of the $41 \mathrm{DA} / \mathrm{M}$ binaries commonly observed by both LAMOST and SDSS, respectively.

We compare the white dwarf parameters of the 27 DA/M binaries and the spectral types of the companions of the 40 systems commonly observed by LAMOST and SDSS in Fig. 6. It is important to emphasize here that the stellar parameters obtained from both the LAMOST and the SDSS DA/M binary spectra rely on the same decomposition/fitting routine. The reliability of this method has been tested by Rebassa-Mansergas et al. (2007); Rebassa-Mansergas et al. (2010), who compared their stellar parameter determinations from SDSS spectra to those obtained by independent spectroscopic parameter fitting methods applied to the same SDSS spectra (Raymond et al. 2003; van den Besselaar et al. 2005; Silvestri et al. 2007; Heller et al. 2009). This comparison revealed that all studies provide stellar parameters that are overall consistent with those obtained from our decomposition/fitting routine (see Rebassa-Mansergas et al. 2007, 2010, for further details). Therefore, directly comparing the stellar parameters obtained here from LAMOST spectra to those obtained from SDSS spectra allows us to test in a direct way how reliable our measured stellar parameters are. Inspection of Fig. 6 reveals that the obtained spectral types are in good agreement (we find that in $\sim 50$ per cent of the cases the spectral types
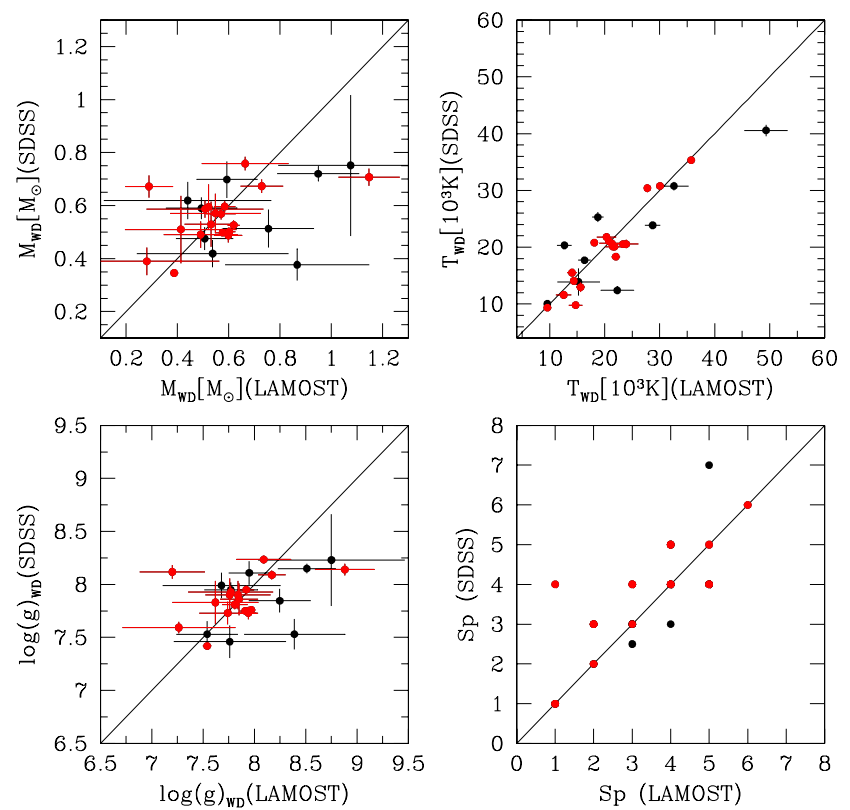

Fig. 6. Comparison of the white dwarf mass (top left), effective temperature (top right), surface gravity (bottom left), and secondary star spectral type (bottom right) obtained for 27 (40 in the bottom right panel) DA/M binaries commonly observed by LAMOST and SDSS. In red are shown systems for which their LAMOST and SDSS spectra have a S/N higher than 12 .

are the same, and that only in two cases the difference is of more than one spectral subclass), and that the white dwarf stellar parameters agree at the $1.5 \sigma$ level in $\sim 70$ per cent of the 


\section{J. J. Ren et al.: WDMS binaries from LAMOST}

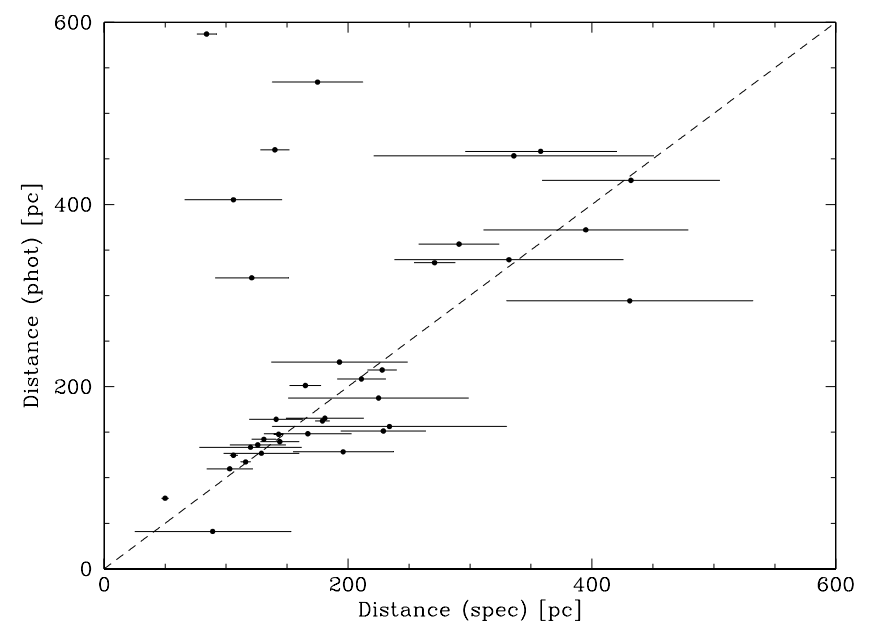

Fig. 7. Photometric vs. spectroscopic distances to 36 LAMOST DA/M binaries with available SDSS DR 8 ugriz magnitudes and LAMOST spectra of $\mathrm{S} / \mathrm{N}$ above 12 .

cases (this percentage drops to $\sim 40$ per cent if we consider the white dwarf effective temperatures; top right panel of Fig. 6). Because systematic uncertainties in the flux calibration affect both the SDSS and LAMOST spectra, it is difficult to assess from which spectra our routine provides the most reliable stellar parameters. However, and not surprisingly, we find the $\mathrm{S} / \mathrm{N}$ of the LAMOST and/or the SDSS spectra to be systematically lower for the cases with the larger discrepancies between the measured values. Indeed, if we consider only DA/M binaries for which the S/N of both the LAMOST and SDSS spectra are higher than 12 (red solid dots in Fig. 6), then the vast majority of cases associated with the larger discrepancies in the measured parameters disappear ${ }^{3}$. We therefore only consider further the stellar parameters of 48 LAMOST DA/M binaries for which the $\mathrm{S} / \mathrm{N}$ of their spectra is higher than 12 .

In order to investigate the effect of the relative flux calibration of the LAMOST spectra in our distance determinations we followed an independent approach for estimating the distances and compared the results. We restricted this exercise to the 48 systems in which the LAMOST spectra are of $\mathrm{S} / \mathrm{N}$ higher than 12 . We interpolated the $T_{\text {eff }}$ and $\log g$ values determined for each white dwarf in these DA/M binaries in the tables by Bergeron et al. (1995) and obtained the white dwarf absolute magnitudes $U G R I Z$. We then gathered available DR 8 ugriz, magnitudes for 36 of our selected targets and simply derive their distances from their distance modulus. Given that the apparent magnitudes correspond to those of the binary system and the absolute magnitudes are calculated for the white dwarf components we considered only the distance obtained from the $u$-band distance modulus in each case, as the M dwarf contribution in this range is generally low. We compare the distances obtained in this way (hereafter photometric distances) to the distances obtained from the flux-scaling factors (hereafter spectroscopic distances) in Fig. 7. Inspection of the figure reveals that the distances are in relatively good agreement except for five cases in which the spectroscopic distances are considerably lower than the photometric distances. In three of these particular cases the DA/M binary spectra are dominated by the contribution of the

\footnotetext{
3 In order to ensure a direct comparison we estimate the $\mathrm{S} / \mathrm{N}$ of the LAMOST and SDSS spectra in the same way as $\frac{1}{n} \sum \frac{f}{e f}$, where $f$ and ef are the flux and flux error respectively, and $n$ is the total number of data points.
}

secondary stars (contributing also with a significant amount of flux in the $u$-band), therefore their photometric distances cannot be considered as reliable. This exercise demonstrates that the relative flux calibration of LAMOST spectra does not seem to affect dramatically our spectroscopic distance determinations and we hereafter adopt these as the distances to our DA/M binaries. These values are included in Table 1. The fact that two independent distance estimates broadly agree for the vast majority of the considered cases provides indirect support for the white dwarf parameters derived from the LAMOST spectra being reliable, as both distance determinations rely to some extent on the accuracy of these parameters.

\section{Characterisation of the LAMOST WDMS binary population}

In this section we study the intrinsic properties of the LAMOST DA/M binary population and investigate whether this new sample helps in overcoming the selection effects incorporated by the target selection criteria of SDSS, which is biased against the identification of systems containing cool white dwarfs and early-type companions. We do this analysing and comparing the stellar parameter and distance distributions of the LAMOST and SDSS DA/M binary populations. However, we note that the 251 SDSS DA/M binaries observed as part of SEGUE were not considered here, as this population was the result of a dedicated search of systems containing systematically cooler white dwarfs and earlier-type companions (Rebassa-Mansergas et al. 2012a). In addition, we only consider in this exercise systems for which the $\mathrm{S} / \mathrm{N}$ of their spectra are higher than 12 (Sect. 4), a restriction we applied both to LAMOST and SDSS DA/M binary spectra.

We begin showing the distance distribution of LAMOST and SDSS DA/M binaries in the top left panel of Fig. 8. To facilitate the comparison both distributions have been normalised. It becomes obvious that, in general, the LAMOST DA/M binary sample contains systems that are considerably nearer to us than those observed by SDSS, i.e. LAMOST observes a smaller and more local volume of DA/M binaries. This is further illustrated in the top right panel of Fig. 8, where we provide the distance cumulative distributions. As we will show below, this difference is likely a consequence of the different observing conditions of the two surveys, i.e. LAMOST DA/M binaries need to be nearer to us for their LAMOST spectra reaching high enough $\mathrm{S} / \mathrm{N}$ for determining reliable stellar parameters. Rebassa-Mansergas et al. (2010) showed that selection effects affect differently the observed population depending on the distance. Thus for example, the fraction of systems containing cool white dwarfs and/or late-type companions increases for decreasing distances (Rebassa-Mansergas et al. 2010, see their Fig. 16). In the same way, DA/M binaries containing hotter white dwarfs and/or earlier type companions dominate at larger distances. Given that (1) due to selection effects the DA/M binary observed population depends on the distance considered and (2) the LAMOST and SDSS DA/M binary distance distributions are considerably different, all SDSS DA/M binaries located at distances larger than $450 \mathrm{pc}$ (the upper limit distance of the LAMOST DA/M binary sample) were not further considered. The total number of LAMOST and SDSS DA/M binaries used in this analysis was thus 48 and 398, respectively.

The middle four left panels of Fig. 8 show the white dwarf parameter and the secondary star spectral type normalised distributions of our 48 LAMOST DA/M binaries. For comparison, we also show the normalised stellar parameter distributions of the 398 SDSS DA/M binaries. In order to compare quantitatively the two populations we applied Kolmogorov-Smirnov (KS) tests to 

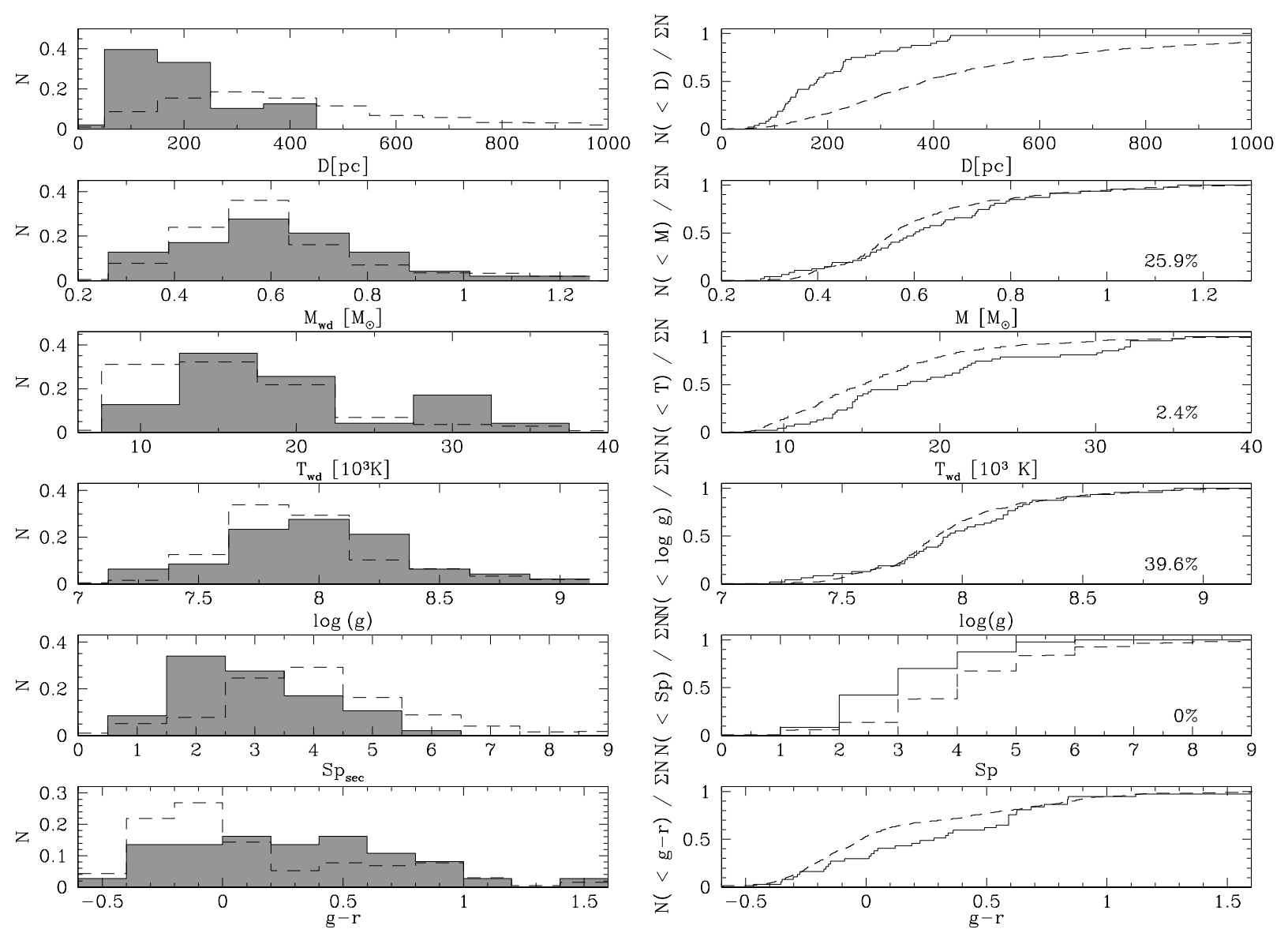

Fig. 8. From top to bottom: normalised distributions (solid line for LAMOST DA/M binaries, dashed line for SDSS DA/M binaries) of distance, white dwarf mass, effective temperature and surface gravity, spectral type of the companion star and $g-r$ colour. Kolmogorov-Smirnov probabilities resulting from comparing the stellar parameter determinations from both samples are indicated in the right panels.

the white dwarf parameter cumulative distributions, and apply a $\chi^{2}$ test to the cumulative spectral type distributions (right panels of Fig. 8). We obtained a $\chi^{2}$ probability of 0 and KS probabilities of 0.02 (effective temperature), 0.39 (surface gravity) and 0.26 (mass).

We mentioned above that, due to selection effects, the observed DA/M binary population depends on the distance considered. Thus, Rebassa-Mansergas et al. (2010) demonstrated that the fraction of SDSS DA/M binaries containing relatively cool $(\lesssim 10000 \mathrm{~K})$ white dwarfs and/or late type ( increases for decreasing distances. This is simple to understand: cool white dwarfs (late companions stars) become too faint to be detected at large distances, however are easily accessible at shorter distances such as those considered here $(\sim 50-450 \mathrm{pc})$. This effect can clearly be seen in the spectral type and white dwarf effective temperature distributions of SDSS DA/M binaries (Fig. 8), which contain a large number of such systems. In contrast, the LAMOST DA/M binary population is dominated by hotter white dwarfs and earlier type companions. This may be a consequence of the different target selection criteria used by the two surveys. However, we have shown that $g-r \gtrsim 0.7$ for $\sim 40$ per cent of LAMOST and $\sim 30$ per cent of SDSS DA/M binaries (Sect. 3.3), typical colours of systems containing cooler white dwarfs and later-type companions (Rebassa-Mansergas et al. 2010). Therefore, the fraction of such systems in LAMOST should actually be larger than in SDSS, which is not the case (see also the bottom panels of Fig. 8). It is important to keep in mind that we are considering in this analysis only LAMOST and
SDSS DA/M binaries with spectra of $\mathrm{S} / \mathrm{N}$ higher than 12 , a condition that seems to exclude a large number of LAMOST DA/M binaries containing cool white dwarfs and late-type companions. We explain this as followed.

The average seeing conditions of Xinglong Observing Station, where LAMOST is located, are considerably worse than in Apache Point Observatory, the observational site of SDSS. Hence larger exposures are needed for LAMOST to reach the same S/N as SDSS would if both surveys observed the same system. However, the exposure times used by LAMOST and SDSS are roughly similar (typical exposure times are 30-90 min, depending on the considered plate), hence the LAMOST spectrum of a given DA/M binary would generally have a lower $\mathrm{S} / \mathrm{N}$ as compared to an SDSS spectrum of the same system. Thus DA/M binaries containing cool white dwarfs and late type companions that are easily observed by SDSS at $\sim 50-450 \mathrm{pc}$ are generally either too faint for LAMOST to be observed, or LAMOST observed them and the resulting spectra are of too low $\mathrm{S} / \mathrm{N}$ for obtaining reliable stellar parameters (i.e. they are excluded from this analysis). This reflects in the peak at M3-4 in the spectral type distribution of SDSS DA/M binaries shifting to M23 for LAMOST DA/M binaries, and in the lack of late-type (>M6) companions. In the same way this effect explains both the scarcity of white dwarfs cooler than $T_{\text {eff }} \lesssim 15000 \mathrm{~K}$ and the pronounced peak at $T_{\text {eff }} \sim 15000-20000 \mathrm{~K}$ in the white dwarf effective temperature distribution of LAMOST DA/M binaries. This naturally explains the null $\chi^{2}$ and very low $(\sim 2.4$ per cent) KS probability obtained when comparing the secondary star 


\section{J. J. Ren et al.: WDMS binaries from LAMOST}

Table 3. Na I $\lambda \lambda$ 8183.27, 8194.81 absorption doublet and $\mathrm{H} \alpha$ emission radial velocities measured for the LAMOST DA/M binaries.

\begin{tabular}{ccccccc}
\hline \hline Name & $\begin{array}{c}\text { HJD } \\
\text { [days }]\end{array}$ & $\begin{array}{c}R V(\mathrm{NaI}) \\
{\left[\mathrm{km} \mathrm{s}^{-1}\right]}\end{array}$ & Err & $\begin{array}{c}R V(\mathrm{H} \alpha) \\
{\left[\mathrm{km} \mathrm{s}^{-1}\right]}\end{array}$ & Err & Telescope \\
\hline J002952.22+434205.8 & 2456266.01670 & 31.90 & 11.58 & 57.23 & 11.69 & LAMOST \\
J002952.22+434205.8 & 2456266.03649 & 35.86 & 12.11 & 50.08 & 11.06 & LAMOST \\
J004432.63+402635.2 & 2455886.07410 & -34.50 & 15.34 & - & - & LAMOST \\
J005221.55+440335.4 & 2456206.22816 & 8.05 & 14.00 & - & - & LAMOST \\
J005221.55+440335.4 & 2456206.24664 & 21.90 & 15.67 & - & - & LAMOST \\
J005221.55+440335.4 & 2456207.14976 & - & - & 32.44 & 11.19 & LAMOST \\
J005221.55+440335.4 & 2456207.16534 & 19.19 & 15.16 & 35.74 & 12.10 & LAMOST \\
J005636.91+383332.6 & 2455913.98067 & -0.61 & 11.73 & - & - & LAMOST \\
J005636.91+383332.6 & 2455914.00629 & 3.49 & 11.90 & - & - & LAMOST \\
J005601.44+400718.4 & 2455886.04888 & -3.34 & 16.11 & - & - & LAMOST \\
\hline
\end{tabular}

Notes. Heliocentric Julian dates and the telescope used for obtaining the spectra (either LAMOST or SDSS) are also indicated. “-” indicates that no radial velocity is available. The complete table can be found at the CDS.

spectral type and white dwarf effective temperature cumulative distributions of both populations (Fig. 8).

Based on the above arguments, LAMOST DA/M binaries may be expected to also contain systematically lower-mass white dwarfs, as low-mass white dwarfs have larger radii and are therefore more luminous (for a given effective temperature). However, even though the fraction of such white dwarfs is slightly larger among LAMOST DA/M binaries, the white dwarf surface gravity and mass distributions are similar for both LAMOST and SDSS systems (Fig. 8). This is further supported by the KS probabilities derived by comparing the corresponding cumulative distributions ( $\sim 26$ per cent for the mass, and $\sim 40$ per cent for the surface gravity distributions). At a given temperature, a $0.4 M_{\odot}$ white dwarf is $\sim 1.3$ times larger than a $0.6 M_{\odot}$ white dwarf (Bergeron et al. 1995; Rebassa-Mansergas et al. 2011), i.e. it has a luminosity $\sim 1.7$ times larger. Conversely, for a given surface gravity (or mass if we keep the radius fixed), the luminosity of a $15000 \mathrm{~K}$ white dwarf is $\sim 5$ times larger than $\mathrm{a} \sim 10000 \mathrm{~K}$ white dwarf. Therefore, the contribution of the effective temperature to the luminosity of a white dwarf is generally much more important than that of its radius. This fact may explain why the population of LAMOST DA/M binaries is not systematically dominated by systems containing low-mass white dwarfs.

We conclude that LAMOST DA/M binaries are statistically different than SDSS DA/M binaries. This is not a consequence of the different target selection criteria of the two surveys, but likely due to the observing site conditions being generally worse at Xinglong Observing Station than at Apache point Observatory, where the LAMOST and the SDSS telescopes are located, respectively. This effect naturally explains why the LAMOST $\mathrm{DA} / \mathrm{M}$ binary population is found between $\sim 50-450 \mathrm{pc}$ and is dominated by systems containing hot white dwarfs and earlytype companions. However, it is important to keep in mind that our conclusion is mainly based on the low KS and $\chi^{2}$ probabilities obtained when comparing the LAMOST and SDSS white dwarf effective temperature and secondary spectral type distributions, respectively. Low KS probabilities may also be obtained as a consequence of different datasets leading to different quality determinations, a possibility we cannot rule out here as the uncertainties in the white dwarf stellar parameters derived from LAMOST spectra are generally larger (see Fig. 6).

\section{Identification of close LAMOST WDMS binaries}

In this section we make use of all available LAMOST and SDSS spectra of our LAMOST DA/M binaries to analyse the fraction of close binaries, i.e. PCEBs, in our sample. We detect close binaries via a radial velocity (RV) analysis based on multi-epoch spectroscopy. If we detect significant (more than $3 \sigma$ ) RV variation we consider the system as a close binary. If we do not detect $\mathrm{RV}$ variation we consider the system as a wide binary candidate. This is because the probability exists for the RVs to sample the same orbital phase of a PCEB, in which case we would not detect RV variation; furthermore, low-inclination and long orbital period PCEBs are more difficult to identify.

We measured the RVs fitting the Na I $\lambda \lambda 8183.27,8194.81$ absorption doublet and/or the $\mathrm{H} \alpha$ emission line of each spectrum following the method described by Rebassa-Mansergas et al. (2008); Ren et al. (2013). This routine fits the $\mathrm{Na}$ I $\lambda \lambda 8183.27,8194.81$ absorption doublet with a second order polynomial plus a double-Gaussian line profile of fixed separation, and the $\mathrm{H} \alpha$ emission line with a second order polynomial plus a single-Gaussian line profile. We were able to obtain at least one RV value for 63 of our LAMOST DA/M binaries, 41 of which have available RVs measured from the $\mathrm{H} \alpha$ emission line, 46 from the Na I doublet, and 22 with RVs from both the $\mathrm{H} \alpha$ emission line and the $\mathrm{Na}$ I doublet. However, only for 42 systems we did obtain at least two $\mathrm{Na}$ I and/or two $\mathrm{H} \alpha$ emission RVs, suitable for the detection of close binaries. The RVs are provided in Table 3.

We detect more than $3 \sigma \mathrm{NaI} \mathrm{RV}$ variation in four systems: J0759+3219, J0958-0200, J1054+5122 and J1649+1412. $\mathrm{J} 0759+3219$ and $\mathrm{J} 1054+5122$ were previously identified as PCEBs by Rebassa-Mansergas et al. (2011); Schreiber et al. (2010). J0958-0200 and J1649+1412 are new PCEBs displaying RV variation from LAMOST spectra obtained during the same night. Therefore, J0958-0200 and J1649+1412 are two excellent candidates for being short orbital period PCEBs. Furthermore, we detect $\mathrm{H} \alpha$ emission $\mathrm{RV}$ variation in 8 systems: the four mentioned above displaying also significant $\mathrm{Na}$ I RV variation, plus J0757+3230, J0819+0604, J1125+2905, J1347+2707. However, the RVs measured from the Na I doublet do not differ significantly for J0757+3230 and J1347+2707. It is worth noting that Rebassa-Mansergas et al. (2008) already pointed out that using $\mathrm{H} \alpha$ emission RVs for identifying PCEBs may not be adequate in some cases, therefore we wish to emphasize that our $\mathrm{H} \alpha$ emission RVs should be considered with caution. In particular, further $\mathrm{Na}$ I RVs are needed to confirm that the PCEBs identified here solely by $\mathrm{H} \alpha$ emission $\mathrm{RV}$ variation are indeed close binaries. This is definitely not the case for J0757+3230 and $\mathrm{J} 1347+2707$.

From the above exercise we find that $4 / 30$, i.e. $\sim 13$ per cent, of our LAMOST DA/M binaries with more than two available 
Na I RVs are PCEBs. If we consider only systems with spectra taken separated by at least one night (otherwise we are biased against the detection of PCEBs of orbital periods longer than $\sim 1$ day; Schreiber et al. 2010) the close binary fraction increases to $2 / 6$, i.e. $\sim 33$ per cent. Although we suffer from low number statistics, this value is similar to the PCEB fraction found among SDSS DA/M binaries ( 21-24 per cent, Nebot Gómez-Morán et al. 2011) and agrees broadly with the $\sim 25$ per cent predicted by population synthesis studies (e.g. Willems \& Kolb 2004). However, we need to take into account that the PCEB fraction depends on the spectral type of the secondary star, as angular momentum loss via magnetic braking is much more efficient in decreasing the orbital separation when the secondary star is partially convective $(\sim \mathrm{M} 0-3)$ than when it is fully convective ( M4-9). Therefore, DA/M binaries containing $\sim \mathrm{M} 0-3 \mathrm{com}-$ panions become semi-detached faster and the PCEB fraction is lower at these spectral types (Politano \& Weiler 2006; Schreiber et al. 2010). The secondary star spectral types of the four systems we have identified as PCEBs are M2 (J0759+3219), M3 (J0958-0200 and J1054+5122) and M4 (J1649+1412). The measured SDSS PCEB fraction at these spectral types increases from $\sim 20$ per cent at M2 to 40 per cent at M4 (Schreiber et al. 2010). Given that the LAMOST DA/M binary population is dominated by systems containing M2-3 secondary stars (Fig. 8), the real PCEB fraction among LAMOST DA/M binaries should not be too far from the value obtained here of $\sim 33$ per cent.

\section{Summary and conclusions}

We have developed an efficient, fast and novel method based on the wavelet transform to identify white dwarf-main sequence (WDMS) binaries containing a DA white dwarf and a M-dwarf companion (DA/M binaries) within the data release 1 of LAMOST. We find 121 of such systems, 80 of which are new discoveries, and estimate our catalogue to contain $\sim 90$ per cent of all DA/M binaries observed by LAMOST. We expect the spectra of the $\sim 10$ per cent of DA/M binaries we missed to lack from important data points in the areas which must be used for the wavelet transform, to be clearly dominated by the flux of one of the two stars, or to be too noisy for a clear identification of the spectral features of both stellar components.

We have applied a decomposition/fitting routine to the LAMOST DA/M binary spectra to measure the white dwarf parameters (effective temperature, surface gravity and mass), obtain the secondary star spectral types, and estimate the distances to our systems. We find that reliable stellar parameters and distances can be obtained for $48 \mathrm{DA} / \mathrm{M}$ binaries having LAMOST spectra of $\mathrm{S} / \mathrm{N}$ above 12 . The stellar parameter and distance distributions are used to analyse the properties of the LAMOST DA/M binary sample and to investigate whether or not this population is statistically different from the SDSS DA/M binary population, which is biased towards the detection of systems containing cool white dwarfs and early-type companions. We find that the LAMOST and SDSS DA/M binary populations are different. However, this is not due to the different target selection criteria of both surveys, but likely a simple consequence of the different observing site conditions, which are generally worse at Xinglong Observing Station than at Apache Point Observatory. Thus, the population of LAMOST DA/M binaries is concentrated at $50-450 \mathrm{pc}$ and is dominated by systems containing hot white dwarfs and early-type companions. Even though LAMOST also fails at identifying systems containing cool white dwarfs, the LAMOST DA/M binary sample dominated by early-type companions is an important addition to the current sample of known spectroscopic SDSS WDMS binaries. Forthcoming LAMOST data releases will allow us to identify more of such systems and to thus build-up the small current sample of LAMOST (DR 1) DA/M binaries.

We have also identified four DA/M binaries in our sample displaying significant radial velocity variation, i.e. PCEBs. Two of these systems were previously identified as close binaries by SDSS, the other two are new PCEBs of expected short orbital periods. We find the fraction of PCEBs among LAMOST DA/M binaries to be $\sim 33$ per cent.

Acknowledgements. This work is supported by the National Key Basic Research of China 2014CB845700 and the National Natural Science Foundation of China under grant numbers 11390371, 11103031, 11233004, and 61273248 . Guoshoujing Telescope (LAMOST) is a National Major Scientific Project built by the Chinese Academy of Sciences. Funding for the project has been provided by the National Development and Reform Commission. LAMOST is operated and managed by the National Astronomical Observatories, Chinese Academy of Sciences. The LAMOST Data Release One Web site is http: //data. lamost. org/dr1/. We thank Detlev Koester for providing us with his grid of white dwarf model spectra, and the anonymous referee for his/her comments and suggestions that helped improving the quality of the paper. A.R.M. acknowledges financial support from the Postdoctoral Science Foundation of China (grant 2013M530470) and from the Research Fund for International Young Scientists by the National Natural Science Foundation of China (grant 11350110496).

\section{References}

Adelman-McCarthy, J. K., Agüeros, M. A., Allam, S. S., et al. 2008, ApJS, 175, 297

Bergeron, P., Wesemael, F., \& Beauchamp, A. 1995, PASP, 107, 1047

Camacho, J., Torres, S., García-Berro, E., et al. 2014, A\&A, 566, A86

Carlin, J. L., Lépine, S., Newberg, H. J., et al. 2012, Res. Astron. Astrophys., 12, 755

Chen, L., Hou, J.-L., Yu, J.-C., et al. 2012, Res. Astron. Astrophys., 12, 805

Chui, C. K. 1992, Wavelets: A tutorial in theory and applications (San Diego: Academic Press)

Cui, X.-Q., Zhao, Y.-H., Chu, Y.-Q., et al. 2012, Res. Astron. Astrophys., 12, 1197

De Marco, O., Passy, J.-C., Moe, M., et al. 2011, MNRAS, 411, 2277

Davis, P. J., Kolb, U., \& Willems, B. 2010, MNRAS, 403, 179

Deng, L.-C., Newberg, H. J., Liu, C., et al. 2012, Res. Astron. Astrophys., 12, 735

Farihi, J., Hoard, D. W., \& Wachter, S. 2010, ApJS, 190, 275

Ferrario, L. 2012, MNRAS, 426, 2500

Heller, R., Homeier, D., Dreizler, S., \& Østensen, R. 2009, A\&A, 496, 191

Iben, I. J., \& Livio, M. 1993, PASP, 105, 1373

Koester, D. 2010, Mem. Soc. Aston. It., 81, 921

Koester, D., Kepler, S. O., Kleinman, S. J., \& Nitta, A. 2009, J. Phys. Conf. Ser., 172,012006

Li, X.-R. 2012, Spectroscopy and Spectral Analysis, 30, 94

Liu, X.-W., Yuan, H.-B., Huo, Z.-Y., et al. 2014, IAU Symp., 298, 310

Luo, A.-L., Zhang, H.-T., Zhao, Y.-H., et al. 2012, Res. Astron. Astrophys., 12, 1243

Marsh, T. R., Parsons, S. G., Bours, M. C. P., et al. 2014, MNRAS, 437, 475

Morgan, D. P., West, A. A., Garcés, A., et al. 2012, AJ, 144, 93

Nebot Gómez-Morán, A., Schwope, A. D., Schreiber, M. R., et al. 2009, A\&A, 495,561

Nebot Gómez-Morán, A., Gänsicke, B. T., Schreiber, M. R., et al. 2011, A\&A, 536, A43

Parsons, S. G., Marsh, T. R., Copperwheat, C. M., et al. 2010, MNRAS, 402, 2591

Parsons, S. G., Marsh, T. R., Gänsicke, B. T., et al. 2012a, MNRAS, 419, 304 Parsons, S. G., Marsh, T. R., Gänsicke, B. T., et al. 2012b, MNRAS, 420, 3281

Parsons, S. G., Marsh, T. R., Bours, M. C. P., et al. 2014, MNRAS, 438, L91

Politano, M., \& Weiler, K. P. 2006, ApJ, 641, L137

Pyrzas, S., Gänsicke, B. T., Brady, S., et al. 2012, MNRAS, 419, 817

Raymond, S. N., Szkody, P., Hawley, S. L., et al. 2003, AJ, 125, 2621

Rebassa-Mansergas, A., Gänsicke, B. T., Rodríguez-Gil, P., Schreiber, M. R., \& Koester, D. 2007, MNRAS, 382, 1377

Rebassa-Mansergas, A., Gänsicke, B. T., Schreiber, M. R., et al. 2008, MNRAS, 390,1635

Rebassa-Mansergas, A., Gänsicke, B. T., Schreiber, M. R., Koester, D., \& Rodríguez-Gil, P. 2010, MNRAS, 402, 620 


\section{J. J. Ren et al.: WDMS binaries from LAMOST}

Rebassa-Mansergas, A., Nebot Gómez-Morán, A., Schreiber, M. R., Girven, J., \& Gänsicke, B. T. 2011, MNRAS, 413, 1121

Rebassa-Mansergas, A., Nebot Gómez-Morán, A., Schreiber, M. R., et al. 2012, MNRAS, 419, 806

Rebassa-Mansergas, A., Zorotovic, M., Schreiber, M. R., et al. 2012, MNRAS, 423, 320

Rebassa-Mansergas, A., Agurto-Gangas, C., Schreiber, M. R., Gänsicke, B. T., \& Koester, D. 2013a, MNRAS, 433, 3398

Rebassa-Mansergas, A., Schreiber, M. R., \& Gänsicke, B. T. 2013b, MNRAS, 429, 3570

Ren, J., Luo, A., Li, Y., et al. 2013, AJ, 146, 82

Richards, G. T., Fan, X., Newberg, H. J., et al. 2002, AJ, 123, 2945

Schreiber, M. R., Gänsicke, B. T., Rebassa-Mansergas, A., et al. 2010, A\&A, 513, L7

Silvestri, N. M., Lemagie, M. P., Hawley, S. L., et al. 2007, AJ, 134, 741

Song, Y.-H., Luo, A.-L., Comte, G., et al. 2012, Res. Astron. Astrophys., 12, 453

Smolčić, V., Ivezić, Ž., Knapp, G. R., et al. 2004, ApJ, 615, L141

Starck, J.-L., Siebenmorgen, R., \& Gredel, R. 1997, ApJ, 482, 1011
Strauss, M. A., Weinberg, D. H., Lupton, R. H., et al. 2002, AJ, 124, 1810

Tremblay, P.-E., Ludwig, H.-G., Steffen, M., Bergeron, P., \& Freytag, B. 2011, A\&A, 531, L19

Tremblay, P.-E., Ludwig, H.-G., Steffen, M., \& Freytag, B. 2013, A\&A, 559, A104

van den Besselaar, E. J. M., Roelofs, G. H. A., Nelemans, G. A., Augusteijn, T., \& Groot, P. J. 2005, A\&A, 434, L13

Webbink, R. F. 2008, in Astrophys. Space Sci. Lib. 352, eds. E. F. Milone, D. A. Leahy, \& D. W. Hobill, 233

Wei, P., Luo, A., Li, Y., et al. 2013, MNRAS, 431, 1800

Willems, B., \& Kolb, U. 2004, A\&A, 419, 1057

Xiang, M. S., Liu, X. W., Yuan, H. B., et al. 2014, MNRAS, submitted

Yanny, B., Rockosi, C., Newberg, H. J., et al. 2009, AJ, 137, 4377

York, D. G., Adelman, J., Anderson, J. E., et al. 2000, AJ, 120, 1579

Zhao, G., Zhao, Y.-H., Chu, Y.-Q., Jing, Y.-P., \& Deng, L.-C. 2012, Res. Astron. Astrophys., 12, 723

Zorotovic, M., \& Schreiber, M. R. 2013, A\&A, 549, A95

Zorotovic, M., Schreiber, M. R., Gänsicke, B. T., \& Nebot Gómez-Morán, A. 2010, A\&A, 520, A86 